\title{
MENTIRAS, INCONSISTENCIAS Y AMBIVALENCIAS Teoría de la acción y análisis de discurso
}

\author{
LIES, INCONSISTENCIES AND AMBIVALENCES \\ Theory of action and discourse analysis
}

\author{
EnRIQUe Martín CRIAdo martincriado@gmail.com \\ Universidad Pablo de Olavide. Sevilla. España
}

\begin{abstract}
Resumen
Muchas prácticas de análisis de discurso parten de dos supuestos: a) la conducta está más determinada por la cultura interiorizada que por la situación; b) el discurso expresa los elementos culturales -actitudes, valores, etc.- que guían la acción. Frente a ello, se defiende que estamos sometidos a múltiples constricciones, a menudo contradictorias, que determinan nuestra acción independientemente de nuestras creencias. Ello provoca que no seamos coherentes, que hagamos y digamos cosas distintas en diferentes situaciones y que adaptemos nuestros discursos y creencias a nuestras prácticas para justificarlas, manipulando estratégicamente la reserva de recursos culturales disponibles. Tener esto en cuenta lleva a centrar el análisis de discurso en el componente estratégico de los discursos y en sus incoherencias y contradicciones.
\end{abstract}

\section{Palabras Clave}

Ambivalencia sociológica; Carrera moral; Disonancia cognitiva; Estrategia discursiva; Legitimidad.

\begin{abstract}
Many practices of discourse analysis take for granted two assumptions: a) behaviour is more determined by interiorized culture than by situation; b) discourse expresses the cultural elements -attitudes, values, etc. - that are the principles of action. In contrast to these assumptions, the article defends that we are subject to numerous and often contradictory constrictions, which determine our action whatever our beliefs. As a consequence, we usually are not coherent; we do and we say different things in different situations and arrange our discourses and beliefs in order to justify our behaviour, handling strategically the stock of available cultural resources. Taking this into account leads us to focus discourse analysis on the strategic component of discourses, as well as on their incoherencies and contradictions.
\end{abstract}

\section{KEYWORDS}

Cognitive Dissonance; Discourse Strategy; Legitimacy; Moral Career; Sociological ambivalence. 


\section{INTRODUCCIÓN}

El ámbito del análisis de discurso sufre una particular escisión ${ }^{1}$. Por un lado, tenemos "analistas del discurso", procedentes en su mayor parte de la lingüística, que inspeccionan exhaustivamente fragmentos reducidos de discurso ${ }^{2}$. Su objetivo es explicar los rasgos -léxicos, gramaticales, argumentativos, etc.- del discurso. Por otro, científicos sociales que utilizan el discurso como materia prima de investigación -entrevistas, grupos de discusión, prensa, documentos escritos, etc. - para indagar un ámbito social: el discurso aquí no es el objetivo principal del análisis, sino un medio para éste.

Los "analistas del discurso" suelen proceder con mucha precaución para revelar hasta el último matiz de sentido de los fragmentos que diseccionan. A cambio, como suelen tener como punto de partida -y como objeto de análisis- el texto, se les plantea constantemente el problema del contexto: ¿cuánta -y qué-información hay que tomar en cuenta para poder comprender todos los matices del texto ${ }^{3}$ ? Este problema se da menos para los científicos sociales: no suelen operar sobre textos aislados. A cambio, suelen abordar grandes cantidades de material discursivo. Esto lleva en muchos casos a prácticas de resumen o selección arbitraria de información (Antaki et al. 2003). El colectivo IOÉ (2010) ha revisado las investigaciones sobre migraciones con grupos de discusión en España: en la mayoría de los casos el "análisis" se limita a resumir y clasificar los enunciados vertidos en el grupo.

Esta extendida práctica no se debe únicamente al hecho de que permita "analizar" grandes cantidades de discurso en poco tiempo. Su difusión también se debe a que engrana con una visión de los discursos y las prácticas muy común, y que denominaré hipótesis parsoniana, pues sintetiza la teoría de la acción de Parsons. Podemos esquematizarla en los siguientes supuestos: a) los sujetos actúan esencialmente a partir de la cultura interiorizada en la socialización; su conducta está más determinada por la cultura —valores, actitudes, etc.- que por la situación; b) su discurso expresaría la cultura interiorizada; por ello, permitiría "comprenderla": hablando, los sujetos expresan los elementos culturales que guían su comportamiento. El discurso sería la clave para "comprender" los comportamientos.

Estos supuestos pueden llevar al análisis de las ideologías como sistemas de ideas que guían la acción, como hacen algunos "análisis críticos del discurso"4. En las ciencias

\footnotetext{
${ }^{1}$ Este artículo se ha beneficiado de las críticas y comentarios de Esperanza Roquero, Pilar Carvajal Soria, Jorge Ruiz Ruiz, Miguel Beltrán y Manuel A. Río Ruiz. A todos ellos les expreso aquí mi agradecimiento.

${ }^{2}$ Es lo que hace la corriente de "análisis conversacional" (Duranti 2000: cap. 8), pero también buena parte del "análisis crítico del discurso" (ver, p. ej., Wodak 2007).

${ }^{3}$ Esta es también la posición del famoso análisis crítico del discurso que, como afirma Widdowson (1998), en muchos rasgos es una versión modernizada de la crítica literaria.

${ }^{4}$ Cf. Van Dijk (1999), cuya concepción de la ideología reproduce numerosos rasgos de la teoría de la acción de Parsons. Así, afirma categóricamente que "las ideas preceden a las acciones" (p. 212, cf. también cap. 7) o que la interacción no sería posible sin una ideología común (cap. 14).
} 
sociales, sin embargo, es más común — gracias a la extensión de ciertos programas informáticos de análisis de textos- una variante peculiarmente perniciosa que procede de la siguiente manera: a) se fragmentan y clasifican los enunciados siguiendo categorías temáticas; b) los argumentos más repetidos bajo cada categoría se convierten en los motivos y/o cultura de los sujetos investigados; $c$ ) se explican el discurso y los actos relatados por esa cultura; d) se explica la cultura por la socialización.

Estos supuestos producen frecuentemente explicaciones circulares (Antaki et al. 2003). Así, podemos encontrar análisis que, tras etiquetar un paquete de discursos como racistas, los expliquen por una cultura racista, a su vez atribuida a una socialización racista: de un dato observado -y descuartizado-, el discurso, se infiere una cultura no observada que circularmente explica el discurso -y actos no observados- y que, a su vez, es explicada por una socialización que tampoco es observada.

Este artículo pretende cuestionar los supuestos de la hipótesis parsoniana sobre acción, cultura y discurso y sacar consecuencias para el análisis sociológico de discursos. Comenzaré defendiendo que la relación entre culturas interiorizadas, discursos y conductas suele ir en sentido contrario al que defiende la hipótesis parsoniana -muchos motivos se elaboran tras las conductas. Analizaré posteriormente la importancia de los desajustes y las ambivalencias para explicar acciones y discursos. Concluiré proponiendo directrices para el análisis sociológico de discursos ${ }^{5}$.

\section{ACTITUDES, DISCURSOS Y CONDUCTAS}

El correlato de la hipótesis parsoniana en psicología es el concepto de actitud: esta sería una valoración o afecto respecto a un objeto, dotada de cierta estabilidad, que guiaría nuestro comportamiento (Pizarro 1998: 116-124; Potter y Wetherell 1987: 43-55). Las actitudes no se pueden observar directamente: se infieren de las declaraciones verbales (habitualmente, respuestas a cuestionarios). La teoría de las actitudes supone una fuerte relación entre verbalizaciones y conductas no verbales.

LaPiere (1934) cuestionó esta relación en un estudio clásico. Entre 1930 y 1932 viajó por Estados Unidos con una pareja china culta: se alojaron en 66 hoteles, pensiones y campings, comieron en 184 restaurantes; solo fueron rechazados en un camping. En general, fueron bien recibidos y atendidos. Seis meses después, LaPiere envió un cues-

\footnotetext{
${ }^{5}$ En lo que sigue me centraré en el análisis sociológico de discursos, esto es, un análisis cuyo objetivo no sea explicar al detalle las características morfológicas, semánticas, retóricas, etc. de los discursos -y sus implicaturas, presuposiciones, etc.-, sino utilizar los discursos para indagar el funcionamiento de ámbitos de relaciones sociales y de las categorías y estrategias simbólicas en juego en los mismos. Asimismo, aunque hable de discursos en general, la mayoría de los argumentos tomarán como ejemplo -y modelo- los discursos producidos en situación de entrevista o grupo de discusión.
} 
tionario a estos establecimientos: respondieron 128; el 91\% contestó que no aceptaría a chinos.

Este estudio inició una sucesión de investigaciones comparando declaraciones verbales con comportamientos. Deutscher (1973) revisó exhaustivamente estos estudios, concluyendo que existe una relación muy débil entre declaraciones verbales y comportamientos -especialmente cuando las situaciones exigen comportamientos contrarios a las creencias expresadas. Muchos autores lo confirman: hacemos y decimos cosas distintas en diferentes situaciones (Pestello y Pestello 2000; Potter y Wetherell 1978).

¿Mentimos habitualmente? Goffman (1969: 6-7) advierte que esta pregunta mezcla varias dimensiones. Primero, la relación del enunciado con los hechos: "veracidad" describe los hechos reales-y "exhaustividad" - expone todo lo ocurrido. Segundo, la relación del locutor con el enunciado: "honestidad" -cree en lo que dice-y "franqueza" -cuenta todo lo que cree relevante. Podemos ser honestos, pero no veraces - contamos hechos falsos que suponemos ciertos - o francos, pero no exhaustivos - desconocemos muchos detalles.

La discrepancia entre actitudes declaradas y comportamientos puede deberse a: a) el sujeto no es honesto; b) es honesto, pero no veraz — desconoce cómo actuará o las razones de su conducta pasada; c) ambos motivos. De momento podemos afirmar, con Goffman (1963; 1971), que la deshonestidad es un rasgo estructural de la interacción cotidiana. No solo porque solemos sacrificar la sinceridad al mantenimiento de las fachadas y el decoro, o porque ser honestos y francos nos conduciría al ostracismo -los enunciados apropiados no suelen ser los veraces. También porque a menudo es imposible ser honestos. Así, pertenecemos a varios "equipos" — de amigos, laborales, familiares, etc. Estos exigen a sus miembros "sinceridad" —comparte con el equipo la información relevante- y "discreción" —no desveles fuera información comprometida. Uno no puede ser sincero y discreto simultáneamente en todos los equipos en que participa: ha de ser forzosamente deshonesto y poco franco.

Todo enunciado es producto parcialmente de la situación de enunciación: los discursos no son simples "expresiones" de opiniones o hechos, sino jugadas interaccionales de presentación de sí. Podemos decir y hacer cosas distintas en diferentes situaciones con total convicción (Goffman 1986). No insistiré en esta idea, ya desarrollada en Martín Criado (1998). Ahora abordaré la otra posibilidad: quizás los sujetos piensen que tienen unas creencias, valores 0 actitudes, pero las constricciones de la situación se impongan sobre aquellas. ¿Cómo podemos investigar esto? Colocando a sujetos con actitudes distintas ante la misma situación.

\section{EL PODER DE LA SITUACIÓN}

Es lo que hicieron Milgram y Zimbardo en sus famosos experimentos. Milgram (1988) mostró que ciudadanos "normales" pueden obedecer las órdenes de aplicar cargas eléc- 
tricas extremas a sus semejantes. Zimbardo (2008) mostró que estudiantes universitarios habitualmente antiautoritarios pueden convertirse en torturadores en una prisión simulada. Milgram y Zimbardo mostraron el poder de la situación. Se puede replicar que son casos extremos - aunque en el experimento de Milgram toda la presión explícita que se ejercía sobre los sujetos para que siguieran aplicando descargas consistía en que el investigador les repitiera "el experimento debe continuar": una presión nimia comparada, por ejemplo, con las del mundo laboral. Pero el poder de la situación se ha documentado en circunstancias mucho más banales: las personas se comportan de forma racista o no dependiendo de la situación -independientemente de sus creencias- 0 firman —o rechazan hacerlo- una petición imitando el comportamiento de un extraño a su lado (Deutscher 1973: 231-258). Darley y Batson (1973) examinaron cómo reaccionaban estudiantes de teología si al ir a dar un sermón a la facultad -algunos sobre la "parábola del buen samaritano"- encontraban por el camino a un hombre andrajoso gimiendo en el suelo: solo se detuvieron a auxiliarle quienes iban con tiempo; el $90 \%$ de quienes iban retrasados a su sermón prosiguió su camino.

No se precisan grandes coacciones para que las situaciones provoquen determinados comportamientos (Beauvois 2008; Zimbardo 2008). En muchos casos, basta el temor a la desaprobación grupal, la premura por "salvar la cara", dejarse llevar por el "ethos" grupal -emociones colectivas que arrastran a los participantes (Collins 2004: parte 1) -, una escalada de pequeñas decisiones - tras conceder un pequeño favor, no se puede negar el siguiente, algo mayor (Joule y Beauvois 2002) - 0 de comportamientos retroalimentándose en círculos viciosos, como los desafíos entre automovilistas (Collins 2004:206).

Dos elementos esenciales contribuyen al poder de las situaciones: la presión social - sanciones y aprobación del grupo-y la visibilidad de los actos. Mientras que el anonimato permite realizar actos contrarios a la moralidad proclamada (Zimbardo 2008: 298-303), la visibilidad del comportamiento obliga a ser más consistente con los valores públicamente proclamados al anticipar que se tendrá que rendir cuentas (Wallace et al. 2005; Pestello y Pestello 2000). La consistencia entre valores proclamados y actos sería efecto en muchas ocasiones, no de la fuerza motriz de valores 0 actitudes, sino del grado de control social ejercido ${ }^{6}$.

La importancia de las constricciones situacionales cuestiona las actitudes que pretenden medir las encuestas: "se pide al encuestado que revele sus opiniones más íntimas sobre un objeto descontextualizado sin relacionarlo con otros, con la certidumbre de que al entrevistador no le importan sus respuestas y de que nadie sabrá qué dijo" (Deutscher 1973: 149). La encuesta de actitudes pretende captar lo que pensaría y haría el individuo liberado de toda constricción o de todo juicio ajeno sobre su comportamiento.

\footnotetext{
${ }^{6}$ Si tenemos en cuenta que podemos mentir con palabras, pero también engañar con actos (Deutscher 1973: 7), la consistencia entre palabras y actos no implica necesariamente sinceridad: uno puede ser consistente en sus declaraciones y acciones sin creer en ellas.
} 
Pero ese individuo no existe: siempre opinamos ante otros y actuamos en situaciones que comportan presiones y constricciones. Captar esta actitud "íntima" sin efectos reales tiene tanto interés como saber qué haría un estudiante de Stanford en una prisión vacía ${ }^{7}$.

\section{SOCIALIZACIONES SECUNDARIAS Y CARRERAS MORALES}

El poder de la situación cuestiona el peso capital que la hipótesis parsoniana le atribuye a la socialización primaria en la explicación de los comportamientos: según esta hipótesis, los contenidos culturales interiorizados en la infancia conformarían los pilares de la personalidad y conducta adultas. Este énfasis en la socialización primaria es solidario de la teoría de la acción parsoniana: si las socializaciones secundarias fueran importantes, las disposiciones se transformarían continuamente y ya no podrían considerarse como motores de la acción. Ahora bien, si tenemos en cuenta el poder de la situación, hemos de reconocer que la socialización primaria no es tan determinante como pretende la hipótesis parsoniana.

Lahire (1998) afirma que las socializaciones secundarias son importantes debido a dos hechos: a) la personalidad conformada en la socialización primaria no es un sistema compacto y coherente, sino fragmentario y maleable, al ser producto de influencias socializadoras muy dispares; $b$ ) el adulto se enfrenta a situaciones y condiciones distintas de las imperantes en su socialización primaria.

En primer lugar, los procesos de socialización implican una diversidad de agentes -familiares, grupos de pares, escuela, etc.- con influencias divergentes, incluso contradictorias (Baek 2010). Esta socialización plural en contextos sociales heterogéneos produce "sujetos plurales", dotados de repertorios diferenciados de esquemas de percepción y acción (Lahire 1998). Aunque algunos esquemas sean más centrales y resistentes al cambio —al ser compartidos por la mayoría de los agentes de socialización significativos-, otros son más moldeables y periféricos y permiten generar comportamientos diversos en situaciones distintas (Martín Criado 2010: 123-142).

En segundo lugar, los sujetos rara vez actúan en situaciones similares a aquellas donde fueron socializados, ya sea por el continuo cambio social o por rupturas en las trayectorias biográficas individuales: emigración, prisión, desclasamiento, desempleo, etc.

Tenemos, así, un doble desajuste: de las disposiciones incorporadas entre sí; entre las condiciones de producción y de funcionamiento de las disposiciones. Este doble

\footnotetext{
${ }^{7}$ La búsqueda de las verdaderas "actitudes" cuando se actúa sin constricciones situacionales es la antítesis de la concepción de sentido común —la personalidad "verdadera" se revelaría "auténticamente" en las situaciones más difíciles- y deriva en absurdos: ¿la verdadera solidaridad se manifestaría cuando no tuviera ningún coste? Esta ausencia de constricciones situacionales provoca que las encuestas de valores ofrezcan una imagen tan virtuosa de la población: aquí "quedar bien no cuesta nada".
} 
desajuste posibilita un amplio juego de las socializaciones secundarias: las dinámicas sociales modifican a los sujetos.

Hemos visto que las situaciones ejercen poderosos efectos en el comportamiento. La ocupación, durante un tiempo prolongado, de posiciones en redes de relaciones sociales -informales o burocratizadas-, con sus particulares exigencias y constricciones, ejerce efectos aún mayores (Wellman 2000; Pizarro 1998). Goffman (1970) mostró que muchos actos aparentemente absurdos de los internados en instituciones psiquiátricas eran efectos de la posición de interno, tal como la definía y gestionaba la organización. Latour y Woolgar (1988: 191-220) muestran la importancia de las coacciones que comporta una posición en una red con su "ciclo de credibilidad" científica: para investigar se precisan instrumental y colaboradores; para obtenerlos, se precisan subvenciones; para obtenerlas se precisa reconocimiento científico, que se logra publicando artículos donde se expongan investigaciones, etc. No importa la motivación inicial del investigador: esta dinámica circular le impone unos comportamientos —bajo sanción de exclusión del campo científico- independientemente de sus creencias.

La importancia de las constricciones de la posición en modelar nuestros comportamientos y creencias desplaza el foco analítico al concepto de "carrera moral" (Becker 2009; Goffman 1970). Este concepto permite analizar la relación dinámica entre las características objetivas de las posiciones y "la secuencia regular de cambios que la carrera introduce en el yo de una persona y en el sistema de imágenes con que se juzga a sí misma y a las demás" (Goffman 1970: 133). La sucesión de acciones de los diversos sujetos implicados modifica paulatinamente la forma de comportarse y de pensar. Cada paso en esta trayectoria abre un abanico de posibilidades y constricciones nuevas que modifica paulatinamente el horizonte de posibles y la personalidad 8 .

El concepto de carrera moral nos invita a estudiar el comportamiento como el resultado de un proceso de modificación de la persona por las posiciones que sucesivamente ocupa. También nos muestra que muchas creencias son posteriores -no anteriores-a las prácticas, como ocurre con las justificaciones que se van aprendiendo en el curso de una carrera desviante (Becker 2009).

\section{DisOnANCIAS, EXCUSAS Y JUSTIFICACIONES}

Cuando la situación lo permite o incentiva, actuamos conforme a nuestras creencias. Pero a menudo las situaciones nos inducen a comportamientos contrarios a ellas. ¿Alteran estos actos nuestras creencias? Según la teoría de la disonancia cognitiva, sí: para restablecer el equilibrio cognitivo, modificamos nuestras representaciones para hacer (2010).

${ }^{8}$ Se puede ver un ejemplo de análisis de la anorexia en términos de carrera moral en Moreno Pestaña 
aceptable 0 deseable el acto realizado. Si aceptamos defender un discurso contrario a nuestras ideas tendemos a aproximar estas a lo argumentado; si aceptamos realizar una tarea aburrida tendemos a encontrarla interesante. Una condición necesaria para tal cambio es que no nos hayamos sentido obligados o fuertemente inducidos a realizar el comportamiento. Sin recompensa o castigo evidentes, nuestra acción parece voluntaria - aunque haya sido provocada por la situación - y nos atribuimos a nosotros mismos el origen de la acción: tendemos a hallar las buenas razones de nuestro proceder (Beauvois 2008; Festinger y Aronson 1982).

La teoría de la disonancia cognitiva nos muestra que la relación entre creencias y actos frecuentemente va en sentido inverso al que supone la hipótesis parsoniana. También evidencia una importante distancia entre las causas de los actos y los motivos expresados. Normalmente ignoramos los procesos que nos llevan a realizar un acto 0 los factores que condicionan nuestras decisiones (Nisbett y Wilson 1977). No obstante, damos motivos o razones: justificaciones que son válidas si nuestros interlocutores las aceptan. Para ello, han de responder a las expectativas de comportamiento culturalmente compartidas y legitimadas: cada sociedad o grupo social tiene sus "vocabularios de motivos" (Mills 1963) a los que recurrimos para explicar —ante otros y ante nosotros mismos- nuestra acción y negociar su sentido.

Pero no siempre relatamos nuestros motivos. A veces realizamos actos contrarios a nuestros valores declarados y no elaboramos ninguna justificación. Se dan razones cuando alguien las pide: cuando el acto es observado y desafía expectativas compartidas, altera los planes de otras personas o se considera desviante, inmoral 0 inapropiado (Orbuch 1997; Scott y Lyman 1968). Ello nos permite explicar una de las principales críticas recibidas por la teoría de la disonancia cognitiva: a menudo las personas realizan actos que contradicen sus creencias declaradas y no buscan reducir la disonancia (Deutscher, Pestello y Pestello 1993: 144; Swidler 1986). Al no verse obligadas a rendir cuentas, pueden mantener compartimentadas distintas creencias 0 esferas de acción sin necesidad de buscar consistencia entre ellas. La consistencia es una exigencia interaccional: somos consistentes cuando anticipamos que tendremos que rendir cuentas de nuestros actos ${ }^{9}$ (Pestello y Pestello 2000).

Cuando damos razones y motivos de nuestros actos no ofrecemos descripciones exactas u objetivas: negociamos nuestra imagen y el sentido de nuestra acción. Si ésta se juzga inapropiada, podemos negar nuestra responsabilidad en el acto - "excusas"- o redefinirlo para presentarlo como legítimo - "justificaciones". Estos relatos han de someterse a las expectativas culturalmente compartidas sobre cómo son las personas y sus motivos. Entre estas, destaca la exigencia de consistencia. Nuestras

\footnotetext{
${ }^{9}$ Ello explica que las investigaciones hallen menor consistencia entre verbalizaciones y comportamientos en situaciones naturales que en experimentales y cuanto mayor tiempo transcurre entre la medición de la actitud y la del acto (Wallace et al. 2005): la situación experimental y la inmediatez entre ambas mediciones hacen a los sujetos más conscientes de la exigencia de consistencia.
} 
sociedades valoran la capacidad de mantener una personalidad coherente en las circunstancias más diversas (Lahire 1998: 24). De ahí que los relatos biográficos tiendan a esta coherencia. Por ello, al entrevistar debemos sustituir la pregunta "¿por qué hizo usted...?" - recibiremos como respuesta un motivo socialmente aprobado consistente con la presentación de sí general- por “¿cómo llegó a ocurrir tal situación?" (Becker 2009b: 85-90).

¿Deberíamos concluir que analizar estos discursos justificatorios no tiene sentido, pues solo encontraríamos fachadas, falseamientos, apariencias? No.

En primer lugar, las explicaciones tienen efectos en la realidad mediante los efectos que generan en su auditorio. Las reacciones ante un acto potencialmente ofensivo dependen más de la justificación que del hecho en sí (Orbuch 1997: 458). La forma de legitimar nuestras acciones determina cómo los demás nos ven y nos tratan. Si los motivos son socialmente aprobados, nos permiten persistir en nuestras acciones o ganar aliados para las mismas; mediante estos relatos se redefine la legitimidad de determinadas prácticas y su aparición se hace más probable, ya que la anticipación de las justificaciones aceptables dirige el comportamiento (Peretti-Watela y Moatti 2006; Mills 1963).

En segundo lugar, la aceptación o rechazo de estos relatos genera consecuencias en el actor. Ensayamos en las conversaciones relatos sobre nosotros y nuestros actos; cuando son aceptados y alentados, se eleva nuestra autoestima - ganamos "energía emocional" - y los convertimos en nuestras verdaderas razones en esa conversación internalizada que es el pensamiento (Collins 2004: 179-184; Condor y Antaki, 2000). Los relatos socialmente aceptados nos sirven para conferir sentido a hechos traumáticos o dolorosos, para superar heridas o desengaños, para persistir en comportamientos potencialmente cuestionables (Orbuch 1997).

Por último, estos relatos nos informan sobre la dinámica de las legitimidades (Alonso 1998: 54-55): un motivo aceptado es un motivo legítimo. Así, hay una enorme diferencia entre una "excusa" - no soy responsable del acto- y una "justificación" -el acto no fue malo-: en el primer caso no se impugna su carácter ilegítimo; en el segundo, sí, y con ello la definición de lo que es legítimo o no. Los vocabularios de motivos socialmente aceptados nos informan sobre la evolución de las legitimidades: ya no se puede justificar asesinar a la esposa por honor o por celos; la "pasión" deja de ser un motivo legítimo cuando los "crímenes pasionales" se convierten en "violencia de género".

Hace tiempo que la sociología de la salud reconoce la importancia de investigar excusas y justificaciones. Cuando las personas incurren en comportamientos potencialmente perjudiciales para su salud -fumar, tener prácticas sexuales desprotegidas, consumir drogas ilegales, etc.-, en lugar de cambiar sus hábitos de vida suelen modificar sus creencias elaborando justificaciones que minimizan el riesgo de sus prácticas. Estos relatos legitiman su práctica ante sí mismos y los demás y permiten persistir en ella sin sentimientos de culpa o ansiedad (Heikkinen, Patja y Jallinoja 2010; Peretti-Watela y Moatti 2006; Rhodes y Cusick 2002). 
Los sujetos elaboran excusas y justificaciones tras realizar prácticas cuestionables. Esta elaboración sería imposible si no encontraran recursos para legitimar sus prácticas en el conjunto de elementos culturales disponibles. Habitualmente, los encuentran. Ello se debe a que la cultura no es el bloque homogéneo e integrado que algunas teorías han sostenido.

\section{ESTRATEGIAS CULTURALES}

La hipótesis parsoniana considera las culturas —la parte simbólica de los entramados de relaciones sociales - como sistemas coherentes: todos sus elementos estarían perfectamente integrados entre sí. Este "mito de la integración cultural" (Archer 1996) ha sido cuestionado recientemente. La concepción de las culturas como sistemas integrados solo se pudo mantener ignorando la historia, las redes de relaciones en que cada cultura está inserta y los conflictos políticos y sociales ${ }^{10}$ (Amselle 1999; Kuper 2001). Frente a ello, se impone analizar el uso estratégico de los elementos culturales en las luchas y conflictos sociales.

Un elemento esencial en esta perspectiva es el concepto weberiano de "legitimidad". El ámbito simbólico supone legitimación o deslegitimación de posiciones, recursos, prácticas... Los sistemas de categorías, las morales, las normas afianzan o socavan jerarquías: dividen la sociedad en grupos, otorgan o niegan derechos, justifican o impugnan desigualdades... Por ello, las representaciones son objeto de luchas y usos estratégicos: "las clasificaciones prácticas están siempre subordinadas a funciones prácticas y orientadas hacia la producción de 'efectos sociales'” (Bourdieu 1985: 87). Archer (1996) desarrolla esta perspectiva: el cambio cultural depende de las relaciones entre el sistema cultural y el sistema social. Por un lado, tenemos sistemas simbólicos que, lejos de estar perfectamente integrados, pueden albergar contradicciones. Por otro, tenemos relaciones - de alianza o conflicto- entre diversos grupos en el sistema social. El cambio o la estabilidad cultural dependen de las relaciones entre ambas esferas. El analista no podría separar el dominio simbólico del sistema de interacción entre grupos sociales: las elaboraciones culturales no se comprenden aisladas de las luchas sociales donde se utilizan.

Desde el momento en que consideramos la "legitimidad" como algo que ejerce una fuerza propia en el mantenimiento o modificación de las relaciones sociales, nos halla-

\footnotetext{
${ }^{10}$ Beltrán (2004: 48-49) nos muestra un buen ejemplo de la relación entre la afirmación de una cultura homogénea e integrada y el "olvido" de los conflictos sociales. Pitt Rivers, en su análisis (de) Un pueblo en la sierra: Grazalema (ed. orig. 1954), excluye de su objeto de estudio la desigualdad entre jornaleros y terratenientes, los conflictos que los oponen e incluso la reciente guerra civil. Ello le permite afirmar "la unidad moral del pueblo" en torno a "los valores fundamentales de la sociedad andaluza" gracias a los cuales "los diferentes elementos de la estructura se mantienen unidos".
} 
mos ante la "dimensión simbólica" de toda relación social como dimensión estratégica. Por ello, las "culturas" no son totalidades integradas, sino configuraciones inestables y heterogéneas en continua transformación. Además, como los elementos culturales de una formación social provienen de fuentes distintas, jamás pueden estar totalmente integrados entre sí, dejando margen de juego a los actores. Esto ha llevado a autores como Amselle a concebir la cultura como una "reserva" de recursos "que los actores sociales movilizan en función de la coyuntura política" (1999: 13).

Esta concepción desplaza radicalmente el foco del análisis de culturas y discursos: en lugar de buscar sistemas simbólicos coherentes e integrados, se trataría de poner en relación sus características con las luchas y estrategias en que se utilizan. El sistema cultural ofrece un repertorio que los distintos grupos pueden utilizar para legitimar sus prácticas o impugnar las de sus oponentes (Blommaert 2005; Wodak 2001; Condor y Antaki 2000).

\section{DeSAJUSTES, AMBIVALENCIAS Y ESTRATEGIAS SIMBólicAS}

Los elementos que hemos visto hasta el momento destacan la importancia de los desajustes en la acción social. Por un lado, los sujetos no tenemos personalidades integradas en torno a una cultura internalizada coherente: somos sujetos plurales, con disposiciones heterogéneas. Por otro, hay un desajuste entre las condiciones en que fuimos socializados y aquellas en que actuamos. Las prácticas cotidianas estarán en función, en parte, de los esquemas culturales incorporados en el pasado, pero también de las coacciones inmediatas que comporte la situación. Las mismas creencias sobre los comportamientos legítimos pueden provenir de fuentes muy distintas, y podemos juzgar como ilegítimos comportamientos que forman parte de nuestra socialización primaria - experiencia habitual cuando hay movilidad social—. Todas estas tensiones producen continuas discordancias entre las prácticas y las creencias declaradas y continuas adaptaciones estratégicas de las creencias - recurriendo al stock disponible de elementos culturales- para legitimar las prácticas.

Subrayemos un hecho esencial: esta discordancia entre prácticas y creencias y entre distintas creencias no es algo extraordinario. Por el contrario, constituye un hecho básico de la estructura social. Merton lo señalaba en su ensayo sobre la ambivalencia sociológica, definida como "las expectativas incompatibles que con carácter normativo se asignan a las actitudes, creencias y comportamientos ligados a un estatus (es decir, una posición social) o a un grupo de estatus en una sociedad" (1980: 19). Merton identificaba seis fuentes principales de ambivalencia en la estructura social: a) las expectativas contradictorias depositadas sobre un mismo cometido social; b) el conflicto entre las normas y expectativas asociadas a las distintas posiciones sociales ocupadas por cada persona; c) el conflicto entre los distintos cometidos asociados a un mismo estatus; d) la existencia de valores culturales contradictorios que exigen a los miembros 
de una sociedad cumplir normas mutuamente excluyentes; e) la disyunción entre las aspiraciones prescritas culturalmente y las posibilidades estructurales de realizarlas; f) la experiencia de haber vivido en distintas sociedades o grupos sociales, con valores diferentes.

Es imposible que alguien escape a todas estas fuentes de ambivalencia. Así, muchas posiciones sociales suponen exigencias parcialmente contradictorias, como ocurre con los docentes, cuyos cometidos de mantener la disciplina, enseñar y examinar suelen hallarse en conflicto, especialmente cuando se enfrentan a alumnados reticentes ${ }^{11}$. De hecho, buena parte del aprendizaje de las competencias requeridas por la posición se refiere a la gestión de las exigencias paradójicas que la misma comporta, debido a la distancia que suele separar su funcionamiento real de su definición oficial. Asimismo, muchas de las aspiraciones prescritas culturalmente son elaboradas por cuerpos de especialistas, como los expertos en bienes de salvación —en el más allá (religiones de salvación) o en el más acá (especialistas en manipulaciones psíquicas o corporales que prometen una vida mejor). Estos tienen interés en hacer necesarios sus servicios: una táctica común es problematizar los comportamientos más comunes y proponer unas vías de salvación de difícil acceso - que requeririan, al final, el concurso de un experto. Cuando estos especialistas logran definir la legitimidad de las prácticas de la población en un ámbito - salud, nutrición, sexualidad, educación, maternidad, etc.-, introducen unas exigencias de salvación poco accesibles para la mayoría ${ }^{12}$. Ello provoca una disyunción constante entre las aspiraciones prescritas culturalmente y las posibilidades estructurales de realizarlas.

Podemos ver un ejemplo de la coexistencia de las diversas fuentes de ambivalencia -y su repercusión en las estrategias simbólicas - en las madres que tienen un trabajo asalariado. Estas -y sus cónyuges - se hallan, en primer lugar, en tensión entre los modelos de género en que fueron socializadas y los nuevos modelos legítimos, menos desigualitarios (Moen y Orrange 2002). Además, sufren las exigencias contradictorias que suponen sus posiciones de madres y de trabajadoras: los horarios que exigen las empresas se oponen a los requisitos de ser "buenas madres" —estar dispuestas a "sacrificarse" por sus hijos, a dedicarse por entero a ellos. A su vez, los discursos elaborados por los especialistas respecto a la educación, la crianza, la alimentación - la salud de los hijos suponen unos estándares tan elevados que resulta imposible alcanzarlos (Perälä-Littunen 2007; Cowdery y Knudson-Martin 2005; Hays 1998). Las madres afrontan todas estas ambivalencias mediante estrategias prácticas, pero sobre todo simbólicas: se redefine la buena maternidad, la buena alimentación o la buena educación en función de las constricciones prácticas (Martín Criado y Moreno Pestaña

${ }^{11} \mathrm{Cf}$. Merchán (2005). Ver también el análisis de Río Ruiz (2010) sobre la ambivalencia de la relación de los docentes con las familias más alejadas de la norma escolar y su repercusión en un discurso oscilante.

${ }^{12}$ Para el interesante caso de la disyunción entre prácticas y discursos sobre televisión e infancia, cf. Callejo (2007). 
2005; Martín Criado, 2004). A ello contribuye el que los consejos de los expertos sean contradictorios entre sí (la cultura no forma un sistema coherente), dejando un margen de maniobra para elegir los que mejor legitimen la prácticas. Así, se redefine la buena maternidad incidiendo en el "tiempo de calidad" o revalorizando las guarderías como espacios donde los niños se socializan mejor que en casa (Johnston y Swanson 2006; Tobío 2005; Tardy 2000).

\section{EL DISCURSO COMO PRÁCTICA ESTRATÉGICA}

Recapitulemos los distintos hilos del artículo. Nos conducen a una teoría de la acción y del discurso opuesta en múltiples puntos a la hipótesis parsoniana.

- La socialización en entornos heterogéneos produce sujetos plurales con disposiciones heterógeneas. Algunas serán más centrales y resistentes al cambio, mientras que otras serán más periféricas y moldeables.

- Nuestros comportamientos son transacciones entre las disposiciones en que fuimos socializados y las constricciones de la situación y las posiciones ocupadas. Estas, mediante las coacciones e incitaciones que ejercen, modifican progresivamente nuestros comportamientos y creencias.

- Este proceso no está exento de ambivalencias, de exigencias contradictorias. Estamos continuamente en tensión: por la ambivalencia entre distintas expectativas de comportamiento, por la oposición entre las constricciones que sufrimos y los ideales que proclamamos, por la distancia entre lo que exige la situación presente y los esquemas en que fuimos socializados en el pasado...

- Intentamos resolver estas tensiones mediante componendas prácticas entre las distintas exigencias. Pero en ocasiones actuamos de forma opuesta a nuestros "valores" y "actitudes". Ello puede llevarnos a compartimentar nuestros actos y discursos -actuamos sin problemas de formas distintas en las distintas situaciones - 0 , cuando nos exigen cuentas, a elaborar excusas y justificaciones manejando estratégicamente el stock de recursos culturales- para negociar la legitimidad de nuestro comportamiento. Estos "motivos" siempre son respuestas a cuestionamientos.

- Los motivos o razones elaborados, cuando son aceptados, tienen consecuencias: permiten persistir en el comportamiento y pueden modificar el ámbito de lo legítimo. Nuestros enunciados no son "expresiones" transparentes de motivos u opiniones: son jugadas interaccionales mediante las que negociamos la legitimidad de nuestras conductas y pensamientos. Pero estas jugadas no tienen por qué ser cínicas; los motivos que elaboramos estratégicamente nos permiten sostener nuestra identidad y autoestima. En términos de Goffman, al exponer nuestros motivos solemos ser honestos, pero no veraces. 
Estos presupuestos nos llevan muy lejos de la concepción del discurso como "expresión" de la cultura internalizada. Intentamos actuar a partir de los esquemas simbólicos interiorizados durante nuestras sucesivas socializaciones. A veces lo conseguimos, pero a menudo se imponen las constricciones y/o ambivalencias de situaciones y posiciones. Entonces manejamos estratégicamente las categorías y esquemas simbólicos para legitimar nuestras prácticas. El análisis de discurso se dirigiría, así, tanto a las principales categorías y esquemas simbólicos a partir de los cuales se definen y piensan las prácticas, instituciones y sujetos, como a su manipulación estratégica en función de las prácticas efectivas.

Esta visión de la acción y el discurso se opone a ciertas prácticas de análisis comunes, como la consistente en: a) ordenar el discurso por temas; b) diferenciar, dentro de cada tema, distintos paquetes de opiniones; c) "explicar" la diferencia de prácticas por la diferencia de opiniones. Esta práctica de análisis confina el discurso a su función referencial: los sujetos se limitarían a relatar hechos y opiniones. Sin embargo, si contemplamos los enunciados como jugadas de presentación de sí, al fragmentar el discurso perdemos una información esencial: el papel que juegan las distintas opiniones en la estrategia simbólica de los locutores (Riessman 1990; Potter y Wetherell 1987: 41). Si esta pérdida puede ser importante al tratar de reconstruir hechos - se cuentan unos u otros según la estrategia de presentación-, es demoledora cuando se pretende reconstituir los esquemas simbólicos de los locutores. Al basar el análisis en los argumentos más repetidos le damos más peso a los más legítimos, relegando aquellos enunciados que nos muestran la tensión entre prácticas y legitimidades. Al amputar los contextos de los enunciados, perdemos un elemento capital: su papel en las estrategias simbólicas de los sujetos; en función de estas - de los oponentes, de lo que está en juego, de la estrategia de presentación de sí...-, las mismas personas pueden defender un argumento 0 el contrario. Al agrupar las opiniones en paquetes coherentes, se anulan todas las inconsistencias y ambivalencias.

Pondré un ejemplo. En una investigación con grupos de discusión, las madres de clases populares sostenían enunciados muy diversos en torno a la intención de adelgazar. La mayoría negaba tener ninguna pretensión de adelgazar -aunque otros enunciados contradecían ese rechazo- y atribuía la "obsesión por la delgadez" a "otras"; si en algún momento se reconocía la intención de adelgazar, se justificaba mencionando razones de salud y negando toda preocupación "estética" - eran "otras las obsesionadas con la estética"-. Un análisis basado en fragmentar el discurso por temas dándole más peso a los argumentos más repetidos llegaría a la siguiente conclusión: la mayoría de estas mujeres solo se preocuparía por adelgazar cuando tuvieran problemas de salud ligados al exceso de peso. Este "análisis" dejaría fuera lo esencial: la ambivalencia entre las obligaciones de la "buena madre" —esta debe ocuparse prioritariamente de sus hijos y "no mirarse demasiado" - y el deseo de evitar un cuerpo estigmatizado. Esta ambivalencia nos explica las constantes contradicciones y vaivenes del discurso - no se puede reconocer claramente el deseo de adelgazar, y este solo sería legítimo por razones de salud-, pero también las prácticas: estas también han de componer con la ambivalen- 
cia, con la tensión entre dos expectativas opuestas - tener un cuerpo no estigmatizado y "no mirarse demasiado"- (Martín Criado 2010b).

El análisis del discurso enfocado a reconstituir los esquemas simbólicos indaga así dos dimensiones básicas: las categorías que estructuran el discurso, y su manipulación estratégica (Blommaert 2005; Condor y Antaki 2000; Potter y Wetherell 1987). El seguimiento minucioso de categorías y estrategias discursivas -los discursos y argumentos a los que se oponen, su variación, sus contradicciones, etc. - nos permite: a) acceder a las ambivalencias en que se hallan estructuralmente los sujetos - a las tensiones entre distintas legitimidades, entre ideales y constricciones prácticas, entre el pasado incorporado y lo que exige el presente, entre lo que se hace y lo que se dice, etc.-; b) analizar las soluciones que se ensayan a estos dilemas - desplazando el ámbito de lo legítimo, forjando o modificando categorías, etc.- y cuya comprensión es crucial, pues transforman categorías y legitimidades, incidiendo a su vez sobre las prácticas -aquellas que logran legitimarse persisten mejor.

Este tipo de análisis aborda las estrategias discursivas de forma muy distinta a la perspectiva que pretende reconstruir los motivos y las culturas que orientan la acción. A ésta sólo le interesaría analizar las estrategias de presentación de sí de los locutores con un propósito: distinguir los enunciados honestos de los deshonestos, eliminar "sesgos" o "falsedades". Su objetivo es buscar el discurso auténtico del sujeto auténtico: sus verdaderos motivos y valores, la cultura coherente que les subyace. La perspectiva que aquí defendemos es muy distinta: no busca ningún sujeto ni cultura auténtica, sino la ambivalencia, la tensión en que necesariamente se mueven los sujetos, sus discursos y sus actos. El discurso no nos interesa como "expresión", sino como "práctica": la presentación de sí que hacen los sujetos en entrevistas o grupos de discusión es una parte de su realidad; sus estrategias discursivas son tan reales como sus otros actos, fueron ensayadas y refrendadas en otras interacciones y tienen efectos reales. Solo analizándolas como prácticas -y no como "expresión" de "motivos"- podemos reconstruir su lugar en el conjunto de prácticas discursivas y extradiscursivas y acceder a las ambivalencias, tensiones y exigencias contradictorias que estas estrategias discursivas intentan conciliar.

Analizar los discursos como prácticas estratégicas no implica que los sujetos sean meros jugadores cínicos y todo discurso mera racionalización. Entre ideas y prácticas hay un doble movimiento. Por un lado, las prácticas son "en parte" función de las ideas o creencias previas. Pero, por otro, estas ideas o creencias se alteran en función de las prácticas efectivas. Los discursos, a su vez, se manejan estratégicamente, pero siempre a partir de un conjunto de esquemas simbólicos y de categorías previo; parafraseando a Marx, los sujetos elaboran sus estrategias discursivas a partir de categorías y esquemas simbólicos que no han elegido.

Podríamos sintetizar así los vaivenes entre esquemas simbólicos, prácticas y discursos: a) los sujetos piensan sus prácticas e intentan realizarlas a partir de un conjunto de esquemas simbólicos y categorías, productos de la historia anterior -individual y colectiva-; b) sus comportamientos se alejan en parte de sus intenciones debido a las cons- 
tricciones de las posiciones que ocupan; c) manejan estratégicamente los esquemas simbólicos y categorías para legitimar las prácticas efectivas. Estos son, por tanto, los tres elementos básicos a abordar en un análisis de discurso: a) categorías y esquemas simbólicos básicos; b) constricciones sobre la acción; c) estrategias discursivas. Para indagarlos, el analista tiene varios medios: a) rastrear las estrategias de presentación de sí y la evolución de la censura estructural; b) situar todo discurso en un espacio de discursos; c) privilegiar en la explicación las constricciones que pesan sobre la acción; d) centrarse en los vaivenes, incoherencias y contradicciones del discurso; e) situar cada opinión de un sujeto en el contexto de sus estrategias simbólicas generales.

\section{A. Rastrear las estrategias de presentación de sí y la evolución de la censura estructural.}

Es necesario considerar todo enunciado como una jugada en la interacción: todo discurso se produce en una situación, frente a determinados interlocutores, donde hay una "censura estructural" (Bourdieu 1985) que pesa sobre lo que se puede y se debe decir (Martín Criado 1991; 1997; 1998). Ello no debe llevarnos a ver los discursos como simples máscaras: los sujetos adaptan sus discursos, pero también sus otras prácticas, en función de la situación, y el analista debe reconstruir este juego de variaciones que nos remite a la variedad de esquemas simbólicos y de constricciones que encuentran los sujetos en las diversas situaciones.

Un importante recurso es la descripción minuciosa de la "censura estructural" de la situación y de las variaciones que produce en el discurso - aspectos que desaparecen cuando el análisis se basa en resúmenes (Potter y Wetherell 1987: 168; Antaki et al. 2003). Así, los grupos de discusión suelen comenzar con los discursos que todos consideran más legítimos; progresivamente se establece una complicidad entre algunos participantes - "no actuamos como sería ideal..."-y se va redefiniendo lo que se puede y lo que se debe hacer. Estas evoluciones no deben llevarnos a ver el discurso inicial como mera máscara que ocultaría al sujeto "verdadero": su concepción de lo legítimo es una parte esencial de su realidad que afecta a sus prácticas y discursos. Por ello, es preciso analizar estas variaciones: nos muestran las legitimidades en juego y sus exigencias contradictorias, las constricciones que se interponen entre lo ideal y lo real, las estrategias simbólicas para afrontar estas tensiones.

\section{B. Situar todo discurso en un espacio de discursos.}

Como vimos, los motivos se elaboran ante cuestionamientos de nuestras conductas. Los discursos se formulan en un espacio de acusaciones y defensas. Todo sujeto se encuentra ante un espacio de posibles discursivos que definen legitimidades y ante el que ha de situarse para justificar sus prácticas. El discurso solo puede entenderse como toma de posición ante otros posicionamientos; todo texto toma elementos prestados de $-y$ 
responde a- otros textos; el análisis siempre ha de tener en cuenta esta intertextualidad (Blommaert 2005; van Eemeren et al. 2000; Alonso y Callejo 1999; Alonso 1998: caps. 3 y 6; Fairclough 1992: cap. 4 y 5). Así, para comprender lo que dicen las madres sobre sus hijos hay que reconstruir el espacio de discursos que definen qué es una buena madre o en qué consiste educar bien. Al situar los discursos en este espacio vemos su aspecto estratégico: las legitimidades frente a las que se posiciona, su utilización de una parte del repertorio simbólico disponible, los argumentos a los que contesta. Al igual que no podemos entender los movimientos de un tenista sin tener en cuenta los de su rival, no podemos comprender las jugadas discursivas de un sujeto sin situarlas en el espacio de posibles discursivos ${ }^{13}$ : todo argumento es un contraargumento.

Aunque este espacio ha de determinarse en cada caso $^{14}$, podemos señalar, en investigaciones mediante entrevistas y grupos de discusión, dos ámbitos concretos a indagar: a) discursos con legitimidad general; b) discursos de los próximos sociales.

En primer lugar, en multitud de ámbitos, tenemos especialistas que definen las prácticas legítimas: médicos, psicólogos, pedagogos, etc. Todo discurso y toda práctica han de posicionarse frente a estos discursos expertos de legitimidad general: aceptándolos, impugnándolos 0 adoptándolos adaptándolos a las circunstancias particulares. Esta apropiación estratégica de los discursos expertos suele verse favorecida por un hecho: el campo de discursos legítimos rara vez forma un conjunto compacto y coherente, sino que ofrece -por las luchas entre expertos por hacerse con las posiciones dominantes en su campo específico- un repertorio de elaboraciones simbólicas diversas, incluso opuestas.

En segundo lugar, los sujetos elaboran sus estrategias simbólicas en sus relaciones sociales cotidianas. Las justificaciones se piden y se elaboran en estos ámbitos donde se negocia el valor de cada persona ${ }^{15}$. Por ello, es esencial situar cada discurso frente al de los próximos sociales ${ }^{16}$.

\footnotetext{
${ }^{13}$ Ruiz (2009) señala tres niveles del análisis de discursos: textual —características del texto—; contextual —situación de interacción y espacio de posibles discursivos-e interpretativo. Según Ruiz, el análisis textual y contextual serían pasos hacia lo esencial, el análisis interpretativo. Nuestra propuesta es que lo esencial del análisis y la "interpretación" consiste en poner en relación el análisis textual —contenido, argumentos, retóricas, categorías, etc.- con el contextual.

${ }^{14}$ Así, el concepto de campo es una valiosa herramienta para analizar textos producidos por profesionales de la producción simbólica, al enfatizar el espacio de competencia donde cada especialista genera sus productos con la vista siempre puesta en los de sus competidores (Martín Criado 2008).

${ }^{15}$ En este punto nos distanciamos de buena parte de las prácticas de Análisis Crítico del Discurso de autores como Fairclough (1992) o Van Dijk (1999). Al partir del concepto gramsciano de hegemonía, se deja fuera del ámbito analítico todo aquello que supuestamente no contribuya al mantenimiento de las relaciones de dominación que el analista se haya propuesto criticar. Para una crítica de estos enfoques, cf. Verschueren (2001) y Widdowson (1998). Blommaert (2005), con su concepto de "policentrismo" - hay múltiples relaciones de dominación entrecruzadas-, tiene un enfoque más cercano al que aquí defendemos.

${ }^{16}$ Ello explica la superioridad del grupo de discusión para investigar los esquemas simbólicos. En una entrevista individual, el entrevistado habla ante el investigador, alguien con estudios universitarios. En el
} 
Es preciso partir de la oposición y el conflicto: las categorías que más importancia revisten para individuos y grupos son las que delimitan entre nosotros (buenos, racionales...) y ellos (malos, irracionales...); los símbolos que más energía emocional movilizan son aquellos que revisten signos contrarios para distintos grupos (Collins 2004; 98 y 179). Por ello, es imprescindible analizar esta estructura de oposiciones y su manejo estratégico - para lo que pueden ser útiles las herramientas de la semiótica estructural.

\section{Privilegiar en la explicación las constricciones que pesan sobre la acción}

El analista habría de estar muy atento a las constricciones que pesan sobre la acción de los sujetos. Como hemos visto, las acciones no se derivan únicamente de las ideas; también se produce el movimiento inverso. Por ello, el analista ha de preguntarse: ¿qué puede forzar a los sujetos a seguir determinadas líneas de acción? ¿Qué constricciones comporta la posición ocupada? Solamente así puede escapar a los argumentos circulares de las explicaciones culturalistas y a los espejismos de los motivos declarados -que suelen convertir, excepto en los casos de las "historias tristes"17, las prácticas realizadas en opciones libremente decididas.

A partir de este reconocimiento de las constricciones, puede preguntarse en qué medida los argumentos que se manejan, las fronteras que se establecen entre categorías, las legitimidades que se defienden o impugnan, sirven como justificaciones de los cursos de acción emprendidos. En otras palabras, la pregunta que ha de mover el análisis es: ¿por qué le conviene decir esto? ¿Qué defiende al establecer estas distinciones? ¿Para qué le sirve este argumento? (Becker 2009b: 195-206). Ello no implica que toda argumentación sea una justificación cínica: solemos creer sinceramente lo que nos conviene creer y a veces seguimos creyendo cosas que no nos conviene creer. Pero hemos de plantear como hipótesis de partida este componente estratégico para evitar la circularidad de las explicaciones culturalistas de los comportamientos.

\section{Centrarse en los vaivenes, incoherencias y contradicciones del discurso}

En la perspectiva de la cultura como un sistema integrado, el analista busca una coherencia subyacente a los distintos elementos simbólicos. De ahí que intente anular las incoherencias o contradicciones (Potter y Wetherell 1987: 39-42). Desde la perspectiva que defendemos, el analista ha de buscar tanto las coherencias entre los distintos ele-

grupo de discusión, el valor de prácticas y discursos se negocia entre próximos sociales en una situación donde la discusión y las críticas provocan una constante oscilación de los discursos.

17 "Si los hechos pasados y presentes de una vida son en extremo lamentables, quizá una persona no pueda hacer nada mejor que demostrar que no le ha cabido culpa en su dramático destino, y su versión apropiada se definirá como una "historia triste" (Goffman 1970: 154). 
mentos simbólicos como sus contradicciones: los sujetos pueden partir de esquemas simbólicos coherentes, pero también toman prestados elementos de distintos paquetes simbólicos y hacen componendas más o menos congruentes -la racionalidad práctica no tiene la coherencia lógica de la racionalidad teórica (Bourdieu 1980). Además, al no derivarse las prácticas únicamente de las creencias, siempre existen inconsistencias entre las distintas prácticas y las distintas creencias.

Los sujetos suelen hallarse en tensión: entre sus prácticas y sus creencias, entre el pasado incorporado y las constricciones de la situación presente, entre distintos paquetes simbólicos para justificar y darle sentido a sus prácticas. Esta tensión, como muestra el concepto de ambivalencia sociológica, es la situación más habitual. Las oscilaciones, contradicciones e incoherencias del discurso constituyen así un elemento esencial del análisis (Blommaert 2005). Analizando estas oscilaciones según los interlocutores, las apuestas en juego, la evolución de la censura estructural de la situación, podemos comprender las tensiones en que se mueven las prácticas cotidianas, los distintos marcos de sentido que se aplican, la ambivalencia entre distintas normas y legitimidades, las estrategias con los repertorios simbólicos disponibles (Martín Criado 2010b).

\section{E. Situar cada opinión de un sujeto en el contexto de sus estrategias simbólicas generales}

Este punto se opone frontalmente al procedimiento más extendido de análisis del discurso: fragmentarlo en paquetes temáticos para comparar por separado las "opiniones" sobre cada tema. Este procedimiento ignora que los discursos, como presentaciones de sí, se modelan en torno a apuestas centrales - qué es educar bien a los hijos, qué es tener conciencia ecológica, etc. - que estructuran la estrategia discursiva. Así, los grupos de discusión sobre alimentación de madres de clases populares que ya he citado en varias ocasiones se estructuraban en torno a una apuesta central: qué es una buena madre. Esta apuesta permitía comprender enunciados que, aislados de ella, permanecerían oscuros: así, las madres que sostenían el discurso más crítico con la contaminación de los alimentos eran las que más utilizaban alimentos industriales. Esta paradoja no se podía comprender desconectándola de la apuesta central en juego: insistiendo en que todo estaba contaminado intentaban descargarse de la acusación de ser malas madres que no alimentan bien a sus hijos -las que elaboran cocina casera también les alimentarían mal, porque todo está contaminado- (Martín Criado 2004).

En lugar de emprender el análisis por parcelas desconectadas, se impone relacionar los enunciados sobre distintos ámbitos a partir de su componente estratégica. La pregunta "¿para qué sirve este enunciado o categoría?" nos lleva a poner en relación enunciados muy diversos con las apuestas centrales de legitimidad en juego. Ello supone un desplazamiento fundamental en la búsqueda de la coherencia de los discursos: en lugar de buscarla en una cultura integrada, hay que buscarla en la posición que se ocupa y en las estrategias por defender lo que se es y lo que se hace. 


\section{CONCLUSIONES}

El auge de la investigación cualitativa en España ha venido acompañado por prácticas de análisis de discurso muy discutibles. La popularización de programas informáticos de análisis de textos ha suplantado la formación teórica ${ }^{18}$ y generalizado un procedimiento que podría sintetizarse en la siguiente receta: "Haga una lista de temas, trocee cada discurso siguiendo la lista, reagrupe los pedacitos de cada tema y resuma o cite los argumentos más repetidos". Esta popular receta despoja a los discursos de las características que le confieren sentido - su carácter estratégico frente a otros discursos en una situación de ambivalencia-, tomando los enunciados como simples descripciones de opiniones o hechos. Este procedimiento solo puede generar banalidades falaces: banalidades, porque se limita a repetir y resumir enunciados; falaces, porque al conceder a todos los enunciados el mismo peso y privilegiar los más reiterados, se limita a replicar las justificaciones más legítimas, confundiendo las causas de la acción con los motivos expresados e ignorando todas las tensiones en que se desenvuelven las acciones y sus relatos.

Esta práctica de análisis va unida a una visión del discurso restringida a su función referencial y a una concepción de la acción como determinada por la cultura interiorizada en la socialización. Frente a ello, hemos defendido recuperar el poder de la situación y la ambivalencia. Los sujetos no actúan solo movidos por valores o actitudes, sino en buena medida por las constricciones de la situación; además, se hallan habitualmente en situaciones de ambivalencia, enfrentados a exigencias contradictorias. Cuando se les piden cuentas, justifican los actos emprendidos y modifican sus creencias.

Esta concepción de la acción implica ver el discurso de otra manera: no como expresión de culturas interiorizadas, sino como jugada para legitimar la propia posición y prácticas frente a aquellos discursos que podrían cuestionarlas. Su análisis importaría, no tanto como medio para acceder a las razones o impulsos de la acción - llámense culturas 0 actitudes-, sino sobre todo con un doble objetivo: a) dilucidar el juego de tensiones y ambivalencias en que se mueven prácticas y discursos - de ahí el énfasis en las contradicciones e inconsistencias, en rastrear las variaciones del discurso según cambia la censura estructural, en privilegiar las constricciones situacionales en la explicación-; b) ver las estrategias simbólicas para legitimar o deslegitimar a los distintos sujetos y sus prácticas - de ahí el énfasis en situar todo discurso en un espacio de discursos y todo enunciado en la estrategia general de presentación de sí.

Los discursos "hacen" cosas: solo tomando como punto de partida del análisis esta dimensión pragmática — preguntándonos por las apuestas en juego tras los enunciados y las categorías- podremos utilizar el discurso para "comprender". Atlas-Ti.

${ }^{18}$ Hasta el extremo de que algunos artículos afirman que su "metodología de análisis de discurso" es el 


\section{REFERENCIAS BibLIOGRÁFICAS}

Alonso, L. E. 1998. La mirada cualitativa en sociología. Madrid: Fundamentos.

Alonso, L. E. y J. Callejo. 1999. "El análisis del discurso: del postmodernismo a las razones prácticas". Revista Española de Investigaciones Sociológicas 88: 37-74.

Amselle, J. L. 1999. Logiques métisses. París: Payot \& Rivages.

Antaki, C., M. Billig, D. Edwards y J. Potter 2003. "El análisis de discurso implica analizar: crítica de seis atajos analíticos". Athenea Digital 3.

Archer, M. S. 1996. Culture and Agency. Cambridge: Cambridge University Press.

Baek, Y. M. 2010. "An integrative model of ambivalence". The Social Science Journal 47: 609-629.

Beauvois, J. L. 2008. Tratado de la servidumbre liberal. Madrid: La Oveja Roja.

Becker, H. 2009. Outsiders. Buenos Aires: Siglo XXI.

Becker, H. 2009b. Trucos del oficio. Buenos Aires: Siglo XXI.

Beltrán, M. 2004. La estructura social. Barcelona: Ariel.

Blommaert, J. 2005. Discourse. A Critical Introduction. Nueva York: Cambridge University Press.

Bourdieu, P. 1980. Le sens pratique. París: Minuit.

Bourdieu, P. 1985. ¿Qué significa hablar? Madrid: Akal.

Callejo Gallego, M. J. 2007. "En medio de fuertes contradicciones sociales". Telos 73:96-99

Colectivo IOÉ. 2010. “¿Para qué sirve el grupo de discusión?: una crítica del uso de técnicas grupales en los estudios sobre migraciones". Empiria 19:73-99.

Collins, R. 2004. Interaction Ritual Chains. Princeton y Oxford: Princeton University Press.

Condor, S. y Ch. Antaki. 2000. "Cognición social y discurso". Pp. 453-489 en El discurso como estructura y proceso, compilado por T. Van Dijk. Barcelona: Gedisa.

Cowdery, R. S. y C. Knudson-Martin. 2005. "The construction of motherhood: Tasks, relational connection, and gender equality”. Family Relations 54: 335-345.

Darley, J. M. y C. D. Batson. 1973. "'From Jerusalem to Jericho': A study of Situational and Dispositional Variables in Helping Behavior". Journal of Personality and Social Psychology 27:100-108.

Deutscher, I. 1973. What We Say / What We Do. Sentiments \& Acts. Glenview, Illinois: Scott, Foresman and Company.

Deutscher, I., F. P. Pestello y H. F. G. Pestello. 1993. Sentiments and Acts. Hawthorne, New York: Aldine de Gruyter.

Duranti, A. 2000. Antropología lingüística. Madrid: Cambridge University Press.

Fairclough, N. 1992. Discourse and Social Change. Cambridge: Polity. 
Festinger, L. y E. Aronson. 1982. "Activación y reducción de la disonancia en contextos sociales", Pp. 281-298, en Estudios básicos de psicología social editado por J. R. Torregrosa y E. Crespo. Barcelona: Hora.

Goffman, E. 1963. Behavior in Public Places. New York: The Free Press of Glencoe.

Goffman, E. 1969. Strategic Interaction. Philadelphia: University of Pennsylvania Press.

Goffman, E. 1970. Internados. Buenos Aires: Amorrortu.

Goffman, E. 1971. La presentación de la persona en la vida cotidiana. Buenos Aires: Amorrortu.

Goffman, E. 1986. Frame Analysis. An Essay on the Organization of Experience. Boston: Northeastern University Press.

Hays, S. 1998, Las contradicciones culturales de la maternidad. Barcelona: Paidós.

Heikkinen, H., K. Patja y P. Jallinoja. 2010. "Smokers' accounts on the health risks of smoking: Why is smoking not dangerous for me?". Social Science \& Medicine 71: 877-883.

Johnston, D. D. y D. H. Swanson. 2006. "Constructing the 'Good Mother': The Experience of Mothering Ideologies by Work Status". Sex Roles 54:509-519.

Joule, R. V. y J. L. Beauvois. 2002. Petit traité de manipulation à l'usage des honnêtes gens. Grenoble: Presses Universitaires de Grenoble.

Kuper, A. 2001. Cultura. La versión de los antropólogos. Barcelona: Paidós.

Lahire, B. 1998. L'homme pluriel. París: Nathan.

LaPiere, R. T. 1934. "Attitudes vs. Actions". Social Forces 13:230-237.

Latour, B. y S. Woolgar 1988. La vie de Laboratoire. París: La Découverte.

Martín Criado, E. 1991, "Del sentido como producción: elementos para un análisis sociológico del discurso". Pp. 187-212 en El pluralismo metodológico en la investigación social, editado por M. Latiesa. Granada: Universidad de Granada.

Martín Criado, E. 1997. "El grupo de discusión como situación social”. Revista Española de Investigaciones Sociológicas 79:81-112.

Martín Criado, E. 1998. "Los decires y los haceres". Papers 56:57-71.

Martín Criado, E. 2004. "El valor de la buena madre. Oficio de ama de casa, alimentación y salud entre las mujeres de clases populares". Revista Española de Sociología 4:93-118.

Martín Criado, E. 2008. "El concepto de campo como herramienta metodológica". Revista Española de Investigaciones Sociológicas 123:11-33.

Martín Criado, E. 2010. La escuela sin funciones. Barcelona: Bellaterra.

Martín Criado, E. 2010b. "Las grandes tallas perjudican seriamente la salud. La frágil legitimidad de las prácticas de adelgazamiento entre las madres de clases populares". Revista Internacional de Sociología 68:349-373.

Martín Criado, E. y J. L. Moreno Pestaña. 2005. Conflictos sobre lo sano. Sevilla: Servicio de Publicaciones de la Consejería de Salud de la Junta de Andalucía. 
Merchán, F. J. 2005. Enseñanza, examen y control. Barcelona: Octaedro.

Merton, R. K. 1980. Ambivalencia sociológica y otros ensayos. Madrid: Espasa-Calpe.

Milgram, S. 1988. "Obediencia a la autoridad”. Pp. 365-382 en Estudios básicos de psicología social, editado por J. R. Torregrosa y E. Crespo. Barcelona: Hora.

Mills C. W. 1963. "Situated actions and vocabularies of motive". Pp. 439-452 en Power, Politics and People. Nueva York: Oxford University Press.

Moen, P. y R. M. Orrange. 2002. "Careers and lives: Socialization, structural lag, and gendered ambivalence". Advances in Life Course Research 7:231-260.

Moreno Pestaña, J. L. 2010. Moral corporal, trastornos alimentarios y clase social. Madrid: Centro de Investigaciones Sociológicas.

Nisbett, R. y T. Wilson. 1977. "Telling more than we can know: Verbal reports on mental processes". Psychological Review 84:231-259.

Orbuch, T. L. 1997. "People's accounts count: The sociology of accounts". Annual Review of Sociology 23:455-78.

Perälä-Littunen, S. 2007. "Gender equality or primacy of the mother? Ambivalent descriptions of good parents". Journal of the Marriage and the Family 69:341-351.

Peretti-Watela, P. y J. P. Moatti. 2006. "Understanding risk behaviours: How the sociology of deviance may contribute? The case of drug-taking". Social Science \& Medicine 63:675-67.

Pestello, F. P. y H. F. G. Pestello. 2000. "Consistency between Sentiments and Acts". Sociological Inquiry 70:61-73.

Pizarro, N. 1998. Tratado de metodología de las Ciencias Sociales. Madrid: Siglo XXI.

Potter, J. y M. Wetherell. 1987. Discourse and Social Psychology: Beyond Attitudes and Behaviour. Londres: Sage.

Rhodes, T. y L. Cusick. 2002. "Accounting for unprotected sex: stories of agency and acceptability". Social Science \& Medicine 55:211-226.

Riessman, C. K. 1990. "Strategic uses of narrative in the presentation of self and illness". Social Science and Medicine 30:1195-1200.

Río Ruiz, M. 2010. "No quieren, no saben, no pueden: Categorizaciones sobre las familias más alejadas de la norma escolar". Revista Española de Sociología 14:85-105.

Ruiz, J. 2009. "Análisis sociológico del discurso: métodos y lógicas" FQS (Forum: Qualitative Sozialforschung) 10: art.26.

Scott, M. B. y S. M. Lyman. 1968. "Accounts". American Sociological Review 33:46-62.

Swidler, A. 1986. "Culture in action: Symbols and strategies". American Sociological Review 51:273286.

Tardy, R. W. 2000. "'But I Am a Good Mom”. Journal of Contemporary Etnography 29:433-473.

Tobío, C. 2005. Madres que trabajan. Dilemas y estrategias. Madrid: Cátedra. 
Van Dijk, T. A. 1999. Ideología. Barcelona: Gedisa.

Van Eemeren, F. H., S. Jacobs, S. Jackson y R. Grootendorst. 2000. “Argumentación”. Pp. 305-334 en El discurso como estructura y proceso, compilado por T. Van Dijk. Barcelona: Gedisa.

Verschueren, J. 2001. "Predicaments of criticism". Critique of Anthropology 21:59-81.

Wallace, D. S., R. M. Paulson, C. G. Lord y C. F. Bond Jr. 2005. "Which Behaviors Do Attitudes Predict? Meta-Analyzing the Effects of Social Pressure and Perceived Difficulty". Review of General Psychology 9:214-227.

Wellman, B. 2000. "El análisis estructural: del método y la metáfora a la teoría y la sustancia". Política y Sociedad 33:11-40.

Widdowson, H. G. 1998. "The Theory and Practice of Critical Discourse Analysis". Applied Linguistics 19:136-151.

Wodak, R. 2001. "The Discourse-Historical Approach". Pp. 63-94 en Methods of Critical Discourse Analysis, editado por R. Wodak y M. Meyer. Londres: Sage.

Wodak, R. 2007. "Pragmatics and critical discourse analysis: A cross-disciplinary inquiry". Pragmatics \& Cognition 15:203-225.

Zimbardo, P. 2008. El efecto Lucifer. Barcelona: Paidós.

EnRIQUe Martín Criado es Doctor en sociología por la Universidad Complutense de Madrid. Profesor de sociología en la Universidad Pablo de Olavide. Miembro del colectivo Denunciemos los abusos patronales (www.abusospatronales.es). Ha publicado libros y artículos sobre metodología y teoría sociología, sociología de la educación, sociología de la juventud, sociología de la familia y sociología del trabajo. Entre sus últimas obras destacan Les deux Algéries de Pierre Bourdieu (Croquant, 2008) y La escuela sin funciones (Bellaterra, 2010).

\section{RECIBIDO: 24/07/2012}

ACEPTADO: 10/04/2013 